\title{
Pour une approche dynamique du fonctionnement cellulaire
}

\author{
Michel Laurent
}

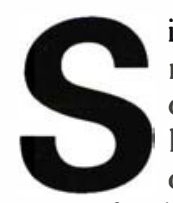
i la combinatoire de quatre nucléotides conduit, via la dégénérescence du code, à l'assemblage de protéines constituées de l'enchaînement de vingt acides aminés, très peu de ces protéines se retrouvent, sous cet état biosynthétique primaire, dans le compartiment cellulaire où s'exerce leur action. La plupart d'entre elles subissent en effet, au cours de leur maturation et en des sites qui leur sont spécifiques, des modifications chimiques catalysées par des enzymes, modifications qualifiées de co-traductionnelles lorsqu'elles se produisent au moment du repliement de la chaîne polypeptidique ou de post-traductionnelles lorsqu'elles se produisent plus tard, une fois la protéine détachée des ribosomes. Les processus de modifications co- et post-traductionnelles des protéines sont si nombreux (plus d'une centaine ont été identifiés à ce jour) et si universellement répandus des bactéries aux eucaryotes, qu'a été évoquée à leur propos l'existence d'un "second code génétique" [1]. A quelques (notables) exceptions près, et en dépit des nombreux travaux consacrés à ce sujet, nous savons peu de choses du rôle de ces modifications, ce qui doit nous amener à réfléchir sur l'idée que nous nous faisons du fonctionnement de la machinerie cellulaire et des moyens mis en œuvre pour aborder cette question.

L'idée centrale que je voudrais développer à partir de l'exemple des modifications co- et post-traductionnelles est la suivante : notre vision du fonctionnement de la cellule est essentiellement binaire ; elle est caractérisée par la recherche de correspondances bijectives entre des états déterminés du système génique et les processus biosynthétiques qui lui sont associés. Les inter-relations entre les premiers et les seconds sont vues au travers d'un cadre de lecture de type anthropomorphique et non comme une correspondance entre l'état initial et l'état final d'une dynamique. Cette vision s'adapte sans doute très bien aux événements se produisant au niveau immédiat des gènes*, si l'on en juge par les formidables succès obtenus par la biologie moléculaire depuis plus de trente ans. Forts de ces succès, nous avons appliqué les techniques de la biologie moléculaire à l'étude de la cellule, en conservant la même approche binaire. Là encore, un certain nombre de réussites spectaculaires ont semblé devoir valider la méthode. Cependant, ces réussites sont de nature beaucoup plus structurelle que fonctionnelle : on démontre que telle protéine possède telles propriétés, interagit avec telle structure ou est impliquée dans tel mécanisme. Cependant, l'invalidation du gène codant pour cette protéine ne produit pas toujours (et même rarement [1] dans le cas où ce gène code pour une enzyme responsable de la modification post-traductionnelle

* Encore que celle remarque mérile quelques nuances pruisque l'on sait aujourd hui que, par exemple, la phosphorylution des facteurs d'initiation el d'elongation joue un rôle important dans ke regulation de la traduction [2]. Ies important dans lu regulation de la traduction [2]. Ies
conditions qui gonvernent l'expression des genes pourraient donc elles aussi être l'expression d'une dynamique. d'une protéine) un changement phénotypique majeur. La raison en est la suivante : si, par analogie, le mécanisme d'expression des gènes correspond à un montage en série, les régulations épigénétiques s'exercent au contraire en parallèle. Quelques précautions et de nouveaux outils doivent être développés pour en comprendre la logique.

Composante spatiale : l'exemple de la spécificité des réactions

Chez les eucaryotes, un et un seul résidu histidine d'une protéine unique (en l'occurrence, le facteur d'élongation EF-2) est modifié de manière post-traductionnelle en diphtamide, la modification mettant en œuvre une séquence de cinq réactions enzymatiques consécutives. Chez la levure, l'invalidation du gène codant pour l'une de ces enzymes [3] ou le remplacement du précurseur His par une variété d'autres résidus [4], n'altèrent pas le caractère fonctionnel de la protéine (elle confère même à la cellule une résistance à la toxine diphtérique dont la cible est précisément le résidu diphtamide). Dans le même ordre d'idées, le facteur d'initiation IF-4D est la seule protéine pour laquelle on ait pu mettre en évidence la transformation d'un résidu lysine en hypusine [5]. Même en considérant qu'un grand nombre des 30000 à 50000 résidus de chaque type présents au sein de l'ensemble des protéines cellulaires sont inaccessibles à la modification, il n'en demeure pas moins que ces observations doivent nous conduire 
imaginer l'existence, dans la cellule, de facteurs ou de conditions liées à l'environnement particulières, à même d'assurer la spécificité de ces réactions.

La réponse de l'enzymologie traditionnelle (in vitro) à la question de la spécificité des réactions est simple : celle-ci résulte d'une complémentarité de structure (pré-existante ou induite) entre l'enzyme et le substrat. L'exemple des protéine kinases et phosphatases [6] montre qu'il n'en va pas nécessairement de même dans un contexte cellulaire. Alors qu'un grand nombre de ces enzymes sont relativement peu spécifiques in vitro, la situation est tout autre dans la cellule grâce à un double mécanisme : (1) l'existence de deux domaines (souvent portés par des sous-unités distinctes) catalytique et régulateur ; (2) une double action de la sous-unité régulatrice qui, d'une part, cible la sous-unité catalytique vers son substrat et, d'autre part, via son interaction avec le site ou la sous-unité catalytique, autorise ou interdit le déroulement de l'acte catalytique.

En d'autres termes, la phosphorylation réversible des protéines met en jeu un mécanisme de découplage entre l'acte catalytique proprement dit et celui de reconnaissance (assurant la spécificité de la réaction) entre l'enzyme et le substrat. Ajoutons qu'il existe une grande diversité de sous-unités régulatrices susceptibles de s'associer à une même sousunité catalytique et pouvant donner lieu à un mécanisme de compétition. Cela signifie que la spécificité de ce type de modification post-traductionnelle ne peut être comprise qu'en incluant, en plus des éléments structuraux, une composante spatiale exprimant la localisation préférentielle de chacun de ces éléments structuraux. Pour d'autres raisons, l'étude de l'acylation des protéines conduit aux mêmes conclusions [7]. Il existe naturellement également une composante temporelle dont l'importance va être soulignée à propos d'un autre type de modification post-traductionnelle, la glycosylation.

\section{Composante temporelle: l'exemple de la glycosylation}

Il est possible de couper par voie en- téines N-glycosylées. Dans ces conditions, l'activité biologique de la plupart de ces protéines mesurée avant et après l'hydrolyse ne présente pas de différences majeures, qu'il s'agisse d'activités enzymatiques ou hormonales [8]. De même, l'extrémité $\mathrm{N}$ terminale d'un certain nombre de récepteurs de facteurs de croissance est normalement glycosylée. Là encore, ces récepteurs sont capables de remplir leur pleine fonction physiologique, qu'ils soient ou non glycosylés. Pour l'un d'entre eux, cependant, on a observé que lorsque sa biosynthèse se déroulait en présence de tunicamycine, inhibiteur de la réaction de glycosylation, le récepteur était inactif. On sait par ailleurs que plusieurs ponts disulfure sont réarrangés au cours du processus de maturation du récepteur. Aussi pense-t-on aujourd'hui que la $\mathrm{N}$-glycosylation de ces protéines a pour objet de donner au précurseur une conformation permettant l'établissement d'une configuration particulière des ponts disulfure, le passage par cette conformation étant une étape obligée du processus de maturation correct du récepteur [9]. Dans ce cas donc, la modification post-traductionnelle remplit une fonction très ciblée dans le temps. Sa mise en évidence a nécessité de prendre en compte la dynamique du processus de biosynthèse et non un simple état final fonctionnel limité à un aspect statique.

Il est impossible de dire, à l'heure actuelle, quel degré de généralité accorder à l'exemple de la glycosylation des récepteurs des facteurs de croissance. Cependant, à la lumière du rôle, de mieux en mieux établi, joué par les protéines chaperons tant dans les processus de repliement que de ciblage des protéines, il est permis de se demander si, dans un certain nombre de cas au moins, le véritable substrat des enzymes de modification co- et post-traductionnelles n'est pas le complexe chaperon-chaîne polypeptidique plutôt que la protéine mûre. Dans ce cas, l'expression de "second code génétique" prendrait alors tout son sens : repliement et ciblage seraient codés par des signaux chimiques spécifiques, renforçant ainsi le déterminisme génétique du fonctionnement cellulaire.

\section{Facteur de couplage: les systèmes} cascades

S'il est nécessaire de considérer une composante spatiale pour comprendre la spécificité d'action, dans la cellule, des protéine kinases et des phosphatases, il est tout aussi important de souligner que la sous-unité servant à la fois d'unité de ciblage et d'unité régulatrice de l'activité enzymatique est, elle-même, la cible de multiples autres kinases et phosphatases. Ce système pourrait permettre à plusieurs voies de régulation de contrôler indirectement l'activité enzymatique du complexe dans lequel se trouve engagée la sous-unité régulatrice et jouer un rôle dans le contrôle de la prolifération cellulaire [6]. Malgré tout, il est là encore insuffisant de s'en tenir à une simple logique alternative si l'on veut appréhender complètement le rôle des mécanismes complexes de phosphorylations/déphosphorylations des protéines dans la cellule. Dans un système cascade, tel que celui-ci, il est impossible de faire l'économie d'une étude dynamique. Le terme de "cascade" s'applique à une situation dans laquelle une enzyme existe sous deux formes interconvertibles douées d'activités spécifiques différentes, l'une modifiée de manière covalente et l'autre non modifiée. La réaction de modification et la réaction inverse de retour à la forme non modifiée sont catalysées par des enzymes distinctes, qui ont donc chacune pour substrat une forme différente de la protéine cible. L'action des protéine kinases et des phosphatases constitue l'exemple type de système cascade. Ajoutons qu'il s'agit de cascades multicycliques puisque chaque protéine kinase ou phosphatase est elle-même susceptible d'être phosphorylée.

La vision commune (mais fausse) que l'on a d'un tel système est parfaitement résumée par la proposition de Krishna et Wold [1] : "En règle générale, les modifications post-traductionnelles réversibles sont des mécanismes servant de commutateurs ON-OFF pour la régulation de l'activité des protéines modifiées." On retrouve une fois encore la logique binaire précédemment évoquée. Si l'analogie du commutateur était correcte, on voit mal, en vertu 
du principe d'économie, pourquoi aurait été sélectionné un processus aussi complexe mettant en ouvre deux réactions enzymatiques distinctes s'exerçant en parallèle mais dans des directions opposées, alors qu'une simple réaction réversible réglée aurait a priori fait l'affaire.

En fait, l'existence en parallèle de deux réactions irréversibles est fondamentale pour le contrôle du flux : cela signifie en effet que sa direction peut être gouvernée par les activités de ces deux enzymes, ce que ne pourrait faire une simple réaction enzymatique réversible (car un catalyseur ne peut modifier la direction d'un flux, celuici étant sous la seule dépendance de paramètres thermodynamiques [10]). I.es propriétés cinétiques in vitro des systèmes cascades ont été décrites il y a maintenant plus d'une quinzaine d'années $[11,12]$. Ces travaux mériteraient aujourd'hui d'être repris et approfondis à la lumière des connaissances acquises depuis sur le mécanisme d'action des protéine kinases et phosphatases. De plus, un certain nombre d'hypothèses simplificatrices devraient nécessairement être abandonnées, dans une optique cellulaire. Il est en particulier peu réaliste de considérer que dans un tel système les enzymes de conversion se trouvent en concentration négligeable par rap port à la protéine substrat. On sait maintenant [13] que l'abandon de cette hypothèse simplificatrice pose de manière nouvelle le rôle des régulations enzymatiques au niveau cellulaire. Néanmoins, deux conclusions majeures de ces études ne devraient pas être remises en cause par ces nouveaux développements : (1) les systèmes cascades sont des intégrateurs de fluctuations métaboliques, cette propriété résultant de leurs capacités d'amplification du signal (ils répondent bien en deçà des constantes de dissociation des effecteurs) ; (2) les cascades de phosphorylation/déphosphorylation des protéines forment un réseau réticulé de réactions réglées par les mêmes messagers secondaires de la cellule (calcium et AMPc principalement). Cette identité constitue très probablement le fondement des processus de synchronisation, donc d'auto-organisation temporelle, d'un grand nombre d'activités cellulaires. Les phénomènes d'auto-organisation, qu'ils soient temporels ou spatiaux, heurtent profondément nos habitudes de pensée. Nous avons tendance à nous représenter l'organisation d'une cellule comme une juxtaposition de compartiments nécessairement matériels, c'est-à-dire séparés par des membranes. Cette vision est assurément trop restrictive, comme nous l'avons montré par exemple à propos des mécanismes de modification post-traductionnelle des microtubules.

\section{Auto-organisation et cohérence à l'échelle supramoléculaire}

En dehors de l'existence d'un noyau et d'un centrosome, l'autre facteur qui caractérise l'émergence de la cellule eucaryote est le développement d'un cytosquelette dont les microtubules constituent l'un des composants majeurs. On dénombre à ce jour cinq modifications post-traductionnelles différentes susceptibles d'affecter les microtubules, modifications dont la fonction reste totalement inconnue, tant chez les métazoaires que chez les protozoaires. Par exemple, la transfection d'un gène modifié d' $\alpha$-tubuline non acétylable chez le flagellé Chlamydomonas n'a permis de mettre en évidence aucun effet phénotypique majeur chez les transformants [14].

Les modifications post-traductionnelles des microtubules présentent une particularité par rapport aux autres mécanismes évoqués jusqu'à présent : les enzymes de modification ont pour substrat les microtubules, c'est-à-dire les polymères de tubuline, tandis que les enzymes assurant la réaction inverse n'agissent, dans la plupart des cas, que sur les formes libres de tubuline. Des contraintes stériques d'accessibilité sont probablement à l'origine de cette spécificité remarquable. L'ensemble des modifications post-traductionnelles conduit à la formation, dans un grand nombre de cellules, de classes différenciées de microtubules, classes dont le rôle fonctionnel reste inconnu. Cependant, au-delà de cet aspect fonctionnel, une question centrale doit être posée : comment s'établit ce répertoire, c'est-à-dire quels mécanismes permettent d'expliquer qu'à un instant et un endroit donnés, la cellule dispose de telle variété de microtubules tandis qu'en un autre endroit ou à un autre instant une autre classe de microtubules est présente?

La réponse conventionnelle à cette question est la suivante : il peut exister une "compartimentation" cellulaire des enzymes de modification et la régulation de la biosynthèse de la tubuline (ou des enzymes de modification) suffit à expliquer la disponibilité d'une classe particulière de microtubules, à un instant donné. Cette explication simple ne résiste pas à l'analyse. Outre le fait que l'invocation d'une compartimentation cellulaire des enzymes de modification ne fait que repousser le problème d'un cran (qu'est-ce qui assure cette compartimentation et comment est-elle maintenue?), elle ne peut rendre compte, par exemple, des résultats obtenus dans l'étude de la spermatogenèse où il a été montré que chaque structure microtubulaire présente une combinaison particulière des différentes formes modifiées, sans que cette combinaison soit liée à une compartimentation d'isotypes [15].

En revanche, la prise en compte de la dynamique des réactions enzymatiques de modification post-traductionnelle des microtubules permet d'apporter une réponse à la question posée. Le point fondamental de toute étude dynamique est de considérer un système ouvert (au sens thermodynamique), c'est-à-dire un système qui échange de la matière et/ou de l'énergie avec le milieu extérieur. Une cellule vivante, comme d'ailleurs chacun de ses sous-ensembles métaboliques, sont par essence des systèmes ouverts. Dans un tel système, il n'existe plus d'état d'équilibre mais un (ou de multiples) états stationnaires. La différence est fondamentale : près de l'équilibre, deux lieux ou deux instants sont équivalents; ils ne le sont plus nécessairement lorsque le système évolue loin de l'équilibre où, comme l'ont montré Prigogine et son école, des brisements de symétrie spatiale et temporelle sont susceptibles de se produire, conduisant à l'apparition de phénomènes cohérents à l'échelle supramoléculaire (structures dissipatives). En considérant un simple processus d'anté-inhibition (générateur d'une non-linéarité) dans l'une des voies de différenciation des microtubules par modification post-traductionnelle, il est possible de montrer [16] que le système va évoluer par 
transitions brutales entre deux états stationnaires distincts caractéristiques d'un basculement entre différentes voies biochimiques de différenciation des microtubules. En d'autres termes, on observera une compartimentation spatiale et/ou temporelle des différentes classes de microtubules sans qu'il existe pour autant de compartiments physiques structurellement séparés.

Il est alors possible de poser le problème de la fonction de manière nouvelle. On a toujours supposé jusqu'ici qu'il fallait rechercher une fonction particulière, de type moléculaire, à chacune des modifications post-traductionnelles des microtubules. L'étude de la dynamique des modifications suggère de renverser cette hypothèse de la manière suivante : les différents isotypes de microtubules sont fonctionnellement équivalents mais sujets à des mécanismes de contrôle différents (au niveau des enzymes de modification) permettant de régler la quantité de microtubules disponibles à un instant et à un endroit donnés. Dans cette optique, la diversité des modifications post-traductionnelles aurait à la fois une origine et une fonction régulatrices. Naturellement, cette proposition n'exclut pas la conception plus traditionnelle et l'on peut penser que l'un ou l'autre des mécanismes est effectif dans un tissu mais pas dans un autre.

\section{Au-delà des modifications post-ura- ductionnelles : Omnis forma ex $A D N$ ?}

Les exemples choisis mettent l'accent sur un contrôle du fonctionnement cellulaire s'exerçant à un niveau épigénétique. Ce contrôle apparaît tout aussi fondamental que celui s'exerçant au niveau des gènes. Les succès de la biologie moléculaire ont en effet quelque peu altéré le sens véritable de la démarche réductionniste. Celle-ci est trop souvent vue comme une tentative de descendre au niveau de description immédiatement inférieur à celui de l'observation. Chaque protéine étant codée par un (ou plusieurs) gène(s), tout processus cellulaire devra obligatoirement se réduire, en dernière analyse, à une interprétation au niveau génique. Cette conception n'a pas plus de sens que celle qui voudrait, par une ré- processus moléculaire une interprétation atomique : dans un cas comme dans l'autre, les fluctuations aléatoires noient les détails des structures les plus fines que l'on est censé considérer. Une approche réductionniste bien comprise [17] doit donc seulement viser à se fonder sur une description d'un niveau pertinent minimum à partir duquel il est possible de reconstruire le phénomène global étudié. Tout le problème va être de définir quel est ce niveau pertinent et il n'est pas certain qu'il s'agisse nécessairement du niveau génique.

La question se pose d'ailleurs dans les mêmes termes en biologie du développement. Ainsi Frankel [18] affirmait-il dès 1989 : "Le dogme selon lequel la formation des patterns dans les organismes pluricellulaires est entièrement gouverné par l'activation différentielle des gènes relève plus de la foi que d'une démonstration. Mon sentiment est que le clonage des gènes et l'étude de la distribution spatiale et temporelle de leurs produits ne conduiront pas à une compréhension exhaustive des mécanismes qui président à la formation des patterns." Cette remarque reste d'actualité dans la mesure où nous ne savons toujours pas quelles sont la nature et les fonctions cibles des gènes homéotiques [19]. La nécessité d'abandonner la doctrine Omnis forma ex $A D N$ a d'ailleurs été reconnue de longue date par les protistologues, plus précisément depuis la découverte par Beisson et Sonneborn [20] de l'existence, chez les ciliés, de phénomènes d'hérédité structurale, c'est-àdire d'une transmission clonale de caractéristiques phénotypiques (inversion de la polarité d'une rangée de cils) sans modification du génome. Ces processus constituent-ils une simple curiosité atypique des protozoaires ? Rien n'est moins sûr, dans la mesure où des processus d'héritage structural ont été plus tard invoqués tant chez les bactéries (maintenance de la polarité des magnétosomes) que chez les mammifères (forme du cytosquelette, forme des cellules, nombre de centres organisateurs des microtubules, etc.) (références citées dans [18]).

En nous montrant que les organismes supérieurs sont capables de diversifier l'expression d'un même message génétique sous forme de plusieurs pro- duits (parfois très différents), la génétique des eucaryotes nous a conduits à repenser la définition du gène et à abandonner le concept "un cistron : une chaîne polypeptidique" au cœur de la génétique bactérienne. Cette diversification de l'expression des messages provient d'une régulation s'exerçant à un niveau post-transcriptionnel via un certain nombre de mécanismes de mieux en mieux établis (choix des sites d'épissage alternatif et processus d'editing du messager, contrôle de son transport du noyau vers le cytosol, etc.). La plupart de ces processus post-transcriptionnels, que l'on classe généralement sous la rubrique "expression des gènes", sont en fait sous contrôle physiologique et ne dépendent pas d'un déterminisme purement génétique [21]. L’important est d'essayer de comprendre, audelà des frontières entre disciplines, comment l'expression d'une dynamique dépendant de facteurs d'environnement (composante physiologique) peut conduire la cellule à effectuer un choix parmi les possibles (composante génétique).

L'insuffisance de la doctrine Omnis forma ex $A D N$ signifie que, dans la cellule, des "conditions locales" doivent, d'une manière ou d'une autre, permettre de moduler les processus d'assemblage, de reconnaissance ou d'interaction. L'identification de ce que sont ces « conditions locales » et la manière dont elles s'expriment en termes de temps, d'espace et de facteurs de couplage, c'est-à-dire dans les termes d'une approche dynamique, apparaissent comme l'un des déf is majeurs que nous pose l'étude du fonctionnement cellulaire. Cette conclusion n'est pas sans conséquence sur les espoirs que fonde la médecine sur la thérapie génique. Il ne fait aucun doute que cette approche permettra d'importantes avancées thérapeutiques. La question est cependant posée de savoir, au-delà de l'établissement de la cartographie du génome, quel pourcentage de maladies et de dysfonctionnements cellulaires pourra être ainsi corrigé. L'identification de la composante génétique d'une maladie ne suffira pas nécessairement à permettre d'en contrôler l'apparition si le produit du gène identifié n'est en eff et qu'un élément intervenant dans une dynamique cellulaire complexe 


\section{RÉFÉRENCES}

1. Krishna R, Wold F. Post-translational modification of proteins. Adv Enzymol 1993 ; 67: 265-98.

2. Sonenberg N. Translation factors as effectors of cell growth and tumorigenesis. Curr Op Cell Biol 1993 ; 5 : 955-60.

3. Mattheakis LC, Shen WH, Collier RJ. DPH5, a methyltransferase gene required for diphtamide biosynthetis in Saccharomyces cerevisiae. Mol Cell Biol 1992 ; 12 : 4026-7.

4. Phan I.D, Perentesis JP, Bodley JW. Saccharomyces cerevisiae elongation factor 2 . Mutagenesis of the histidine precursor of diphtamide yields a functional protein that is resistant to diphteria toxin. J Biol Chem 1993; 268: 8665-8.

5. Cooper HL, Park MH, Folk JE, Safer B, Braverman $R$. Identification of the hypusine-containing protein $\mathrm{Hy}^{+}$as translation initiation factor elF-4D. Proc Natl Acad Soi USA $1983 ; 80: 1854-7$.

6. Keryer G, Bailly E. Spécificité de l'action des protéine kinases et phosphatases dans la cellule. médecine/sciences $1994 ; 10$ : 408-16.

7. Blenis J, Resh MD. Subcellular localization specified by protein acylation and phosphorylation. Curr Op Cell Biol 1993 ; 5 : 984-9.
8. Hart GW, Haltiwanger RS, Holt GD, Kelly WG. Glycosylation in the nucleus and cytoplasm. Annu Rev Biochem 1989; 58 : 841-74.

9. Olson TS, Lane MD. A common mechanism for post-translational activation of plasma membrane receptors. FASEB J $1989 ; 3$ 1618-24.

10. Cornish-Bowden A. Fundamentals of enzyme kinetics. I.ondres: Butterworths, 1979' 116.

11. Stadtman ER, Chock PB. Superiority of interconvertible enzyme cascades in metabolic regulation: analysis of monocyclic systems. Proc Natl Acad Sci USA $1977 ; 74$ : 27615.

12. Chock PB, Stadtman ER. Superiority of interconvertible enzyme cascades in metabolic regulation: analysis of multicyclic systems. Proc Nall Acad Sci USA $1977 ; 74$ : 2766 70.

13. Laurent M, Kellershohn N. Apparent cooperativity for highly concentrated Michaelian and allosteric enzymes. / Mol Biol 1984 ; $174: 543-55$.

14. Kozminski K, Diener DR, Rosenbaum JL. High level expression of nonacetylable Q-tubulin in Chlamidomonas reinhardtii. Cell Motil Cyloskel 1993 ; 25 : 158-70.

15. Fouquet JP, Eddé B, Kann MI, Wolff A, Desbruyeres E, Denoulet P. Differential distribution of glutamylated tubulin during spermatogenesis in mammalian testis. Cell Motil Cyloskel 1994 ; 27 : 49-58.
16. Iaurent $M$, Fleury, A A dynamical mo del for post-translational modifications of microtubules. FEBS Lell 1993 ; 336 : 1-7.

17. Danchin A. Placage et modèles totalitaires. In : Delattre P, Tellier M, eds. Elabo ration et justification des modeles. Applications en biologie. Paris : Maloine, 1979 : 39-56.

18. Frankel J. Pattern formation. Ciliates studies and models. New York : Oxford University Press, 1989 : 259

19. Botas J. Control of morphogenesis and differentiation by HOM/Hox genes. Curr op Cell Biol 1993; 5 : 1015-22.

20. Beisson I Sonneborn TM. Cytoplasmic inheritance of the organization of the cell cortex in Paramecium aurelia. Proc Nall Acad Si USA $1965 ; 5.3$ : 275-82.

21. Hinnebusch A, Hochstrasser M. Posttranscriptional processes. Curr Op Cell Biol $1993 ; 5: 941-3$.

\section{Michel Laurent}

Directeur de recherche au CNRS, service d'imagerie cellulaire, Bâtiment 441, Université Paris-Sud, 91405 Orsay Cedex, France.

\section{TIRÉS À PART}

M. L.aurent. 